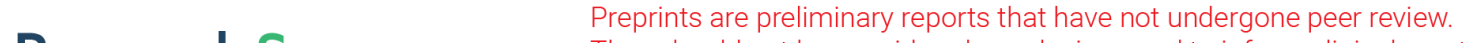 They should not be considered conclusive, used to inform clinical practice, or referenced by the media as validated information. \\ Correlations obtained from optical spectra of Fe- pnictides using an extended Drude-Lorentz model
}

\section{Seokbae Lee}

Sungkyunkwan University

Yu-Seong Seo

Sungkyunkwan University

\section{Seulki Roh}

Sungkyunkwan University

\section{Dongjoon Song}

National Institute of Advanced Industrial Science and Technology

Hirosh Eisaki

National Institute of Advanced Industrial Science and Technology

Jungseek Hwang ( $\square$ jungseek@skku.edu )

Sungkyunkwan University

\section{Research Article}

Keywords: correlation spectra, BaFe2As2, cuprates and Fe-pnictides, narrow coherent and broad incoherent, electron systems

Posted Date: July 19th, 2021

DOI: https://doi.org/10.21203/rs.3.rs-701960/v1

License: (c) (1) This work is licensed under a Creative Commons Attribution 4.0 International License.

Read Full License 


\title{
Correlations obtained from optical spectra of Fe-pnictides using an extended Drude-Lorentz model
}

\author{
Seokbae Lee ${ }^{1}$, Yu-Seong Seo ${ }^{1}$, Seulki Roh ${ }^{1}$, Dongjoon \\ Song $^{2,3}$, Hirosh Eisaki ${ }^{2}$, and Jungseek Hwang ${ }^{1 *}$ \\ ${ }^{1}$ Department of Physics, Sungkyunkwan University, \\ Suwon, Gyeonggi-do 16419, Republic of Korea \\ ${ }^{2}$ National Institute of Advanced Industrial Science \\ and Technology, Tsukuba 305-8568, Japan \\ ${ }^{3}$ Department of Physics and Astronomy, \\ Seoul National University, Seoul 08826, Republic of Korea
}

(Dated: July 10, 2021)

\begin{abstract}
We introduce an analysis model, an extended Drude-Lorentz model, and apply it to Fe-pnictide systems to extract their electron-boson spectral density functions (or correlation spectra). The extended Drude-Lorentz model consists of an extended Drude mode for describing correlated charge carriers and Lorentz modes for interband transitions. The extended Drude mode can be obtained by a reverse process starting from the electron-boson spectral density function and extending to the optical self-energy, and eventually, to the optical conductivity. Using the extended Drude-Lorentz model, we obtained the electron-boson spectral density functions of $\mathrm{K}$-doped $\mathrm{BaFe}_{2} \mathrm{As}_{2}$ (Ba-122) at four different doping levels. We discuss the doping-dependent properties of the electron-boson spectral density function of K-doped Ba-122. We also can include pseudogap effects in the model using this new approach. Therefore, this new approach is very helpful for understanding and analyzing measured optical spectra of strongly correlation electron systems, including high-temperature superconductors (cuprates and Fe-pnictides).
\end{abstract}

${ }^{*}$ Correspondence to [email: jungseek@skku.edu]. 


\section{Introduction}

Fe-pnictide superconductors have been intensively studied since their discovery ${ }^{1}$. They are known as multiband systems because they have multiple orbitals at the Fermi level ${ }^{2-6}$. Compared with single-band copper oxide superconductors, multiband systems may exhibit interesting features such as multiple superconducting gaps ${ }^{3,4,7-10}$ and non-trivial gap symmetries $^{11}$. Fe-pnictide systems are also known as correlated electron systems ${ }^{12}$. This is because the measured optical spectra reveal multiband superconducting gaps ${ }^{7,13}$ and correlations between electrons ${ }^{12,14-16}$. We note that the same analysis method, which has been used to extract information on correlations from measured optical spectra of cuprates ${ }^{17}$, has been approximately applied to extract the electron-boson spectral density function from measured optical spectra of Fe-pnictides ${ }^{14-16}$. An analysis of the optical spectra of multiband Fe-pnictide systems in the normal state also has been performed using two (narrow coherent and broad incoherent) Drude modes ${ }^{10,18-21}$. In the latter analysis, researchers used the two Drude modes to describe the charge carriers of a multiband system in the normal state. Further, there is another method for extracting the electron-boson spectral density function from the measured optical spectra of Fe-pnictide superconducting systems in the superconducting state by using two parallel transport channels ${ }^{22}$. In the case of the analysis with two Drude modes, the correlation effects might be implicitly included in the two Drude modes. In general, the correlation may divide the optical spectral weight of charge carriers into coherent and incoherent components ${ }^{23}$. Therefore, the narrow Drude mode may contain most of the coherent components, while the broad Drude mode may contain most of the incoherent components. However, information on the correlations cannot be obtained explicitly from the aforenoted analysis with the two Drude modes.

In this paper, we develop a new method to explicitly obtain the information on correlations in correlated electron systems. First, we introduce an extended Drude mode, which can be defined based on the extended Drude model formalism ${ }^{24,25}$. This extended Drude mode can be employed to describe correlated charge carriers; therefore, it can be used to explicitly reveal correlation effects from the measured optical spectra of correlated electron systems, including Fe-pnictide systems. In this study, we obtain extended Drude modes from input electron-boson spectral densities using an inverse process that has been introduced and used for analyzing measured optical spectra ${ }^{22,26}$. To simulate measured optical conductivity, 
additional Lorentz modes are added to describe interband transitions generally situated in the high-energy region. Using this new approach, we extract the electron-boson spectral density functions of $\mathrm{K}$-doped $\mathrm{BaFe}_{2} \mathrm{As}_{2}$ (Ba-122) at various doping levels. We compare the results obtained using the extended Drude mode with those obtained using the two Drude modes. Furthermore, we obtain the doping-dependent mass renormalization factor (or correlation strength) from the extracted electron-boson spectral density function. Particularly, we observe a dome-shaped mass renormalization factor as a function of doping. This factor is different from that of cuprates, which monotonically increases as the doping decreases. We demonstrate that this difference can be associated with pseudogaps, which may exist in Fe-pnictide superconducting systems ${ }^{27,28}$.

\section{Analysis models}

We briefly describe the two models used in this study: One is a two Drude (TD)-Lorentz (or TD-Lorentz) model, which consists of two Drude modes for describing intraband transitions (or itinerant charges) and Lorentz modes for interband transitions. In the TD-Lorentz model, optical conductivity can be described as

$$
\begin{aligned}
\tilde{\sigma}(\omega) & =\tilde{\sigma}_{T D}(\omega)-\sum_{k} i\left(\frac{\Omega_{k, p}^{2}}{4 \pi}\right) \frac{\omega}{\omega^{2}-\omega_{k}^{2}+i \omega \gamma_{k}}, \\
\tilde{\sigma}_{T D}(\omega) & =\sum_{i=1}^{2} i\left(\frac{\Omega_{i, D p}^{2}}{4 \pi}\right) \frac{1}{\omega+i \tau_{i, i m p}^{-1}}
\end{aligned}
$$

where $\tilde{\sigma}(\omega)$ is the complex optical conductivity, $\tilde{\sigma}_{T D}(\omega)$ represents the two Drude (TD) modes, and $\Omega_{i, D p}$ and $\tau_{i, i m p}^{-1}$ are the plasma frequency and the impurity scattering rate of $i$ th Drude mode $(i=1$ or 2$)$, respectively. $\omega_{k}, \Omega_{k, p}^{2}$, and $\gamma_{k}$ are the resonance frequency, the strength, and the damping parameter of the $k$ th Lorentz mode. In general, one of the Drude modes is called a narrow (or coherent) Drude and the other is termed a broad (or incoherent) one ${ }^{10,15}$. This analysis model, which is legitimate, has been applied for various multiband systems ${ }^{10,15,18,21,29}$ and spawned many interesting findings including a hidden non-Fermi liquid behavior in $\mathrm{Ba}_{0.6} \mathrm{~K}_{0.4} \mathrm{Fe}_{2} \mathrm{As}_{2}{ }^{10}$.

The other analysis model is an extended Drude-Lorentz (ED-Lorentz) model. Here, we replace the two Drude modes $\left(\tilde{\sigma}_{T D}(\omega)\right)$ with an extended Drude mode $\left(\tilde{\sigma}_{E D}(\omega)\right)$. Therefore, the ED-Lorentz model consists of an extended Drude mode for describing the correlated 
charge carriers and Lorentz modes for interband transitions. The extended Drude mode can be obtained from an input electron-boson spectral density function $\left(I^{2} B(\omega)\right)$ using a reverse process ${ }^{26}$. Here $I$ is the coupling constant between an electron and a force-meditating boson and $B(\omega)$ is the boson spectrum. The reverse process consists of a series of steps starting from $I^{2} B(\omega)$, obtaining the optical conductivity, and eventually, to the reflectance spectrum $^{26}$. For a more detailed description of the reverse process, the readers can refer to Ref. ${ }^{26}$. To get the optical conductivity of the extended Drude mode, we start from an input $I^{2} B(\omega)$ for the extended Drude mode, get the imaginary part of the optical self-energy of the extended Drude mode $\left(-2 \Sigma_{E D, 2}^{o p}(\omega)\right)$ or the optical scattering rate of the extended Drude mode $\left(1 / \tau_{E D}^{o p}(\omega)\right)$ using the generalized Allen formula ${ }^{30,31}$, which can be described as $-2 \Sigma_{E D, 2}^{o p}(\omega) \equiv 1 / \tau_{E D}^{o p}(\omega)=(\pi / \omega) \int_{0}^{\infty} d \Omega I^{2} B(\Omega)\{2 \omega \operatorname{coth}(\Omega / 2 T)-(\omega+\Omega) \operatorname{coth}[(\omega+\Omega) / 2 T]+$ $(\omega-\Omega) \operatorname{coth}[(\omega-\Omega) / 2 T]\}$, where $T$ is the absolute temperature. Then, we calculate the real part of the optical self-energy of the extended Drude mode $\left(-2 \Sigma_{E D, 1}^{o p}(\omega)\right)$ using the KramersKronig relation as the real and imaginary parts of the self-energy form a Kramers-Kronig pair $^{26,32}$, which can be written as $-2 \Sigma_{E D, 1}^{o p}(\omega)=-(2 \omega / \pi) \int_{0}^{\infty} d \omega^{\prime}\left[-2 \Sigma_{E D, 2}^{o p}\left(\omega^{\prime}\right)\right] /\left(\omega^{\prime 2}-\omega^{2}\right)$. Eventually, we obtain the complex optical conductivity of extended Drude mode using the extended Drude model formalism ${ }^{25}$, which can be written as

$$
\tilde{\sigma}_{E D}(\omega) \equiv i\left(\frac{\Omega_{p, E D}^{2}}{4 \pi}\right) \frac{1}{\omega+\left[-2 \tilde{\Sigma}_{E D}^{o p}(\omega)\right]},
$$

where $\tilde{\sigma}_{E D}(\omega)$ is the extended Drude mode and $-2 \tilde{\Sigma}_{E D}^{o p}(\omega)$ is the corresponding complex optical self-energy to the extended Drude mode. $\Omega_{p, E D}^{2} / 8$ is the total spectral weight of the charge carriers (or ED mode) in a correlated material system, where $\Omega_{p, E D}$ is the plasma frequency of the extended Drude mode. Therefore, the measured optical conductivity can be described by the following model:

$$
\tilde{\sigma}(\omega)=\tilde{\sigma}_{E D}(\omega)-\sum_{k} i\left(\frac{\Omega_{k, p}^{2}}{4 \pi}\right) \frac{\omega}{\omega^{2}-\omega_{k}^{2}+i \omega \gamma_{k}} .
$$

It is worth to be noted that the optical self-energy $\left(-2 \tilde{\Sigma}^{o p}(\omega)\right)$ corresponding to the total optical conductivity $(\tilde{\sigma}(\omega))$ can be defined, based on the extended Drude model formalism, as $-2 \tilde{\Sigma}^{o p}(\omega) \equiv i \frac{\Omega_{p}^{2}}{4 \pi} \frac{1}{\tilde{\sigma}(\omega)}-\omega$. In general, the real and imaginary part of the total optical selfenergy $\left(-2 \tilde{\Sigma}^{o p}(\omega)\right)$ do not form a Kramers-Kronig pair. However, the real and imaginary parts of the optical self-energy of the ED mode $\left(-2 \tilde{\Sigma}_{E D}^{o p}(\omega)\right)$ self-consistently form a KramersKronig pair ${ }^{26,32}$. We will discuss this further in the discussion section. 


\section{Results and discussions}

We investigate $\mathrm{K}$-doped Ba-122 $\left(\mathrm{Ba}_{1-x} \mathrm{~K}_{x} \mathrm{Fe}_{2} \mathrm{As}_{2}\right)$ single crystals at four different doping levels namely $x=0.29,0.36,0.40$, and 0.51 , which have the superconducting transition temperatures $\left(T_{c}\right)$ of $35.9 \mathrm{~K}, 38.5 \mathrm{~K}, 38.5 \mathrm{~K}$, and $34.0 \mathrm{~K}$, respectively. The sample at $x=$ 0.40 is optimally doped. The K-doped single crystal samples are grown using a self-flux technique $^{33}$. We take reflectance spectra $\left(35-8000 \mathrm{~cm}^{-1}\right.$ or $\left.\sim 4 \mathrm{meV}-1.0 \mathrm{eV}\right)$ of the four single crystal samples at various temperatures using an in-situ gold evaporation technique ${ }^{34}$ and a continuous liquid helium flow cryostat. In this study, we focus on the measured optical spectra in the normal state $(T=50 \mathrm{~K})$. We obtained the optical conductivity spectra from the measured reflectance spectra using a Kramers-Kronig (KK) analysis ${ }^{35,36}$. For the KK analysis, we take extrapolations to zero frequency and infinity. For the extrapolation to zero frequency, we use the Hagen-Rubens relation $(1-R(\omega) \propto \sqrt{\omega})$. For the extrapolation to infinity, we use an available reported data ${ }^{13}$ up to $40,000 \mathrm{~cm}^{-1}, R(\omega) \propto \omega^{-1}$ from 40,000 to $10^{6} \mathrm{~cm}^{-1}$, and the free electron response $\left(R(\omega) \propto \omega^{-4}\right)$ above $10^{6} \mathrm{~cm}^{-1}$. We show the measured reflectance spectra and the corresponding optical conductivities in Fig. 1.

We use the two (TD-Lorentz and ED-Lorentz) models to analyze the optical conductivity up to $950 \mathrm{meV}$. In Fig. 2, we compare the results obtained by applying the two models to the optical conductivity (at $50 \mathrm{~K}$ ) of the optimally K-doped Ba-122. Fig. 2(a) and 2(b) show the data and fits obtained using the TD-Lorentz and ED-Lorentz models, respectively, below $950 \mathrm{meV}$. We also separately show the two Drude modes, extended Drude mode, and Lorentz modes. We used the same Lorentz modes for both the fittings. The overall quality of fits was similar for the two models. Fig. 2(c) shows the electron-boson spectral density function $\left(I^{2} B(\omega)\right)$ obtained using the ED-Lorentz model. Here, we use a model $I^{2} B(\omega)$ that consists of two Gaussian functions: a sharp Gaussian function and a broad Gaussian function. We used the reverse process to obtain the extended Drude mode from this input electron-boson spectral density function ${ }^{26}$. There are six fitting parameters for $I^{2} B(\omega)$, which consists of two Gaussian functions, and one for $\Omega_{p, E D}$. By adjusting the seven fitting parameters, we obtained a reasonable fit in the low-frequency region, as shown in 2(b). In Fig. 2(d), we show the optical conductivities of both the Drude modes and the ED Drude mode; the two optical conductivities agree well. This result indicates that, in normal state, one may not need to consider two separate transport channels to describe charge carriers in 
the multiband systems. We speculate that the band characteristics are fuzzed by thermal excitations and/or correlations. However, to expose a hidden non-Fermi-liquid behavior, the two-Drude approach is useful, as reported in the literature ${ }^{10,21}$. For superconducting state, a two-channel approach must be used because the multiple bands provide superconducting gaps of different sizes, as reported in the literature ${ }^{3,22}$.

The correlation effects can be estimated from the obtained $I^{2} B(\omega)$. The mass renormalization factor $(\lambda)$ caused by the correlations can be estimated from $I^{2} B(\omega)$ using the well-known formula, $\lambda \equiv 2 \int_{0}^{\omega_{c}}\left[I^{2} B(\omega) / \omega\right] d \omega$, where $\omega_{c}$ is the cutoff frequency. In our case, $\omega_{c}$ is $100 \mathrm{meV}$ because $I^{2} B(\omega)$ is negligibly small above $100 \mathrm{meV}$. The estimated mass renormalization factor of this sample is 1.41. From this quantity, one can obtain separate spectral weights for the coherent and incoherent components ${ }^{23}$. The coherent and incoherent components correspond to $1 /(1+\lambda)$ and $\lambda /(1+\lambda)$ fractions of the total spectral weight of charge carriers, respectively. Specifically, the coherent and incoherent components account for $41 \%$ and $59 \%$ of the total spectral weight of the charge carriers for this optimally doped sample $(x=0.40)$, respectively.

We apply the ED-Lorentz model to the two underdoped $(x=0.29$ and 0.36$)$ and one overdoped $(x=0.51)$ samples. We also used two (sharp and broad) Gaussian functions for $I^{2} B(\omega)$. The underdoped samples may have psuedogaps ${ }^{27,28,37,38}$; nevertheless, for now, we do not include pseudogap in our model. In a later paragraph, we include the pseudogaps in the analysis to show pesudogap effects on the resulting $I^{2} B(\omega)$ (see Fig. 5). In Fig. 3, we show data and fits of four K-doped Ba-122 samples, including the optimally doped sample $(x=0.40)$, at $50 \mathrm{~K}$. The overall shapes of the real parts of optical conductivities of the four samples are similar: a sharp increase below $50 \mathrm{meV}$, a broad shoulder below $200 \mathrm{meV}$, and a broad absorption peak near $500 \mathrm{meV}$. These are the generic features of the optical conductivity of Fe-pnictides ${ }^{10,20,39,40}$. The quality of fits was similar for all four samples. The ED mode and two Lorentz modes are displayed separately for each sample. The plasma frequencies of the extended Drude modes are 18800, 17200, 19200, and $16300 \mathrm{~cm}^{-1}$ for $x=$ $0.29,0.36,0.40$ and 0.51 , respectively. We observed slight doping-dependent evolutions of the ED mode and the Lorentz modes. From these fittings, we obtained the electron-boson spectral density functions $\left(I^{2} B(\omega)\right)$ of the four samples. For the fittings, we did not need to adjust the broad Gaussian peak in $I^{2} B(\omega)$ for all four samples. Therefore, we used the same broad Gaussian peak for all samples, as shown in Fig. 4. 
In Fig. 4, we show the obtained electron-boson spectral density functions $\left(I^{2} B(\omega)\right)$ of our four samples. For $\mathrm{Bi}_{2} \mathrm{Sr}_{2} \mathrm{CaCu}_{2} \mathrm{O}_{8+\delta}$ (Bi-2212), the energy of the sharp peak is proportional to the superconducting transition temperature $\left(T_{c}\right)^{41}$, and its intensity decreases rapidly in the overdoped region ${ }^{17}$. The obtained $I^{2} B(\omega)$ of K-doped Ba-122 also exhibits some doping dependencies. The peak intensities of underdoped and optimally doped samples are similar, but that of the overdoped sample is significantly reduced, similar to the case of Bi-2212; however, the peak position does not show a systematic change with doping. In the inset, we show the mass renormalization factors $(\lambda)$ of the four samples at different K-doping levels. The mass renormalization factor (solid hexagon) shows a dome shape, which is different from that of Bi-2212; the $\lambda$ of Bi-2212 monotonically decreases as the doping increases ${ }^{42}$. This dome-shaped renormalization factor looks similar to a peaked London penetration depth observed around the optimal doping in P-doped Ba-122 43 . However, we expect that these different doping-dependent behaviors of $\lambda$ of two material systems might be related to the pseudogaps. As we mentioned earlier, Fe-pnictide systems have been known to contain the pseudogaps. The pseudogaps exhibit similar temperature- and doping-dependent behaviors of those in cuprates ${ }^{44,45}$. So far, in our analysis model, we did not include the pseudogaps. In general, if pseudogaps are included, the $I^{2} B(\omega)$ spectrum, including the sharp peak, will be shifted to a lower energy ${ }^{46}$, resulting in an increase in the mass renormalization factor.

To demonstrate the pseudogap effect on the electron-boson spectral density function, we include the pseudogap (PG) in the analysis for the two underdoped samples $(x=0.29$ and 0.36). In this case, the optical scattering rate of the extended Drude mode can be written in a more generalized form ${ }^{47}$ as $1 / \tau_{E D}^{o p}(\omega)=(\pi / \omega) \int_{0}^{\infty} d \Omega I^{2} B(\Omega) \int_{-\infty}^{+\infty} d z[N(z-\Omega)+N(-z+$ $\Omega)]\left[n_{B}(\Omega)+f(\Omega-z)\right][f(z-\omega)-f(z+\omega)]$, where $n_{B}(\omega)$ and $f(\omega)$ are the Bose-Einstein and Fermi-Dirac distribution functions, respectively, and $N(z)$ is the normalized density of states, which can be used to describe the pseudogap. The pseudogap is modelled ${ }^{46,48}$ as $N(z)=N_{0}+\left(1-N_{0}\right)\left(z / \Delta_{p g}\right)^{2}$ for $|z|<\Delta_{p g},\left(5-2 N_{0}\right) / 3$ for $|z| \in\left(\Delta_{p g}, 2 \Delta_{p g}\right)$, and 1 for $|z| \geq$ $2 \Delta_{p g}$, where $N_{0}$ is the normalized density of states at the Fermi level and $\Delta_{p g}$ is the sise of the speudogap. We note that, in this PG model, the density of states loss in the pseudogap is recovered just above the pseudogap ${ }^{48,49}$ as shown in the insets of Fig. 5. We assume that the same size of pesudogap $\left(\Delta_{p g}\right)$ is $\sim 28.4 \mathrm{meV}$ for the two samples and the depths $\left(1-N_{0}\right)$ of the pseudogap are 0.9 and 0.5 for $x=0.29$ and 0.36 , respectively. Fig. 5(a) and (c) show the data and fits of the two underdoped samples. We used the same plasma frequencies of 
the extended Drude modes as we used for the cases of previous fittings without including the PG. The new electron-boson spectral density functions, $I^{2} B(\omega)$, obtained including the PG are shown in Fig. 5(b) and (d). We clearly observe the sharp Gaussian peak shifts to a lower energy. The amount of the shift increases as the strength (or depth) of pseudogap increases as reported in a published paper ${ }^{46}$. The new renormalization factors are 1.87 and 1.78 for $x=0.29$ and 0.36 , respectively, as shown in the inset of Fig. 4. The new renormalization factor monotonically increases as the doping decreases, which is a similar doping-dependent behavior of the renormalization factor of hole-doped cuprates ${ }^{42}$. In this study, we mainly focused on the introduction of the extended Drude-Lorentz model approach. Furthermore, we also included the pseudogap in the analysis and obtained some interesting results. However, there might be some complicated issues regarding the pseudogaps for Fe-pnictides, such as the depth (or strength) and energy scale of the pseudogaps.

The ED-Lorentz model, when considered exclusively, does not appear to be completely new because the Drude mode is simply replaced with the extended Drude mode in the prevalent Drude-Lorentz model. However, this approach has not been applied thus far to analyze multiband Fe-pnictide superconducting systems and single-band cuprates. In this regard, the proposed ED-Lorentz model is a novel and effective method for analyzing the optical spectra of correlated electron systems, including high-temperature superconductors. Herein, we describe the extended Drude mode more in detail: Similar to the case of the simple Drude mode, the real and imaginary parts of the extended Drude mode form a Kramers-Kronig pair. In the case of an ideal system that can be described with an extended Drude mode, the real and imaginary parts of the corresponding optical self-energy form a Kramers-Kronig pair as well ${ }^{26,32}$. Moreover, if we include additional Lorentz modes to realize a real correlated electron system, which exhibits both intraband and interband (optical) transitions, then the real and imaginary parts of the total (ED plus Lorentzian) optical conductivity still form a Kramers-Kronig pair holding the causality condition ${ }^{35}$. However, in general, the real and imaginary parts of the corresponding total optical self-energy can no longer form a Kramers-Kronig pair; this is because the optical self-energy with multiple components is related to the optical conductivity with multiple components in the extended Drude formalism. In this sense, optical-self energy is not a completely well-defined optical quantity for describing the measured optical spectra. However, if only extended Drude mode can be extended by excluding all Lorentz modes from the measured optical spectra, 
the optical self-energy of the remaining extended Drude mode can be a well-defined optical quantity. Interestingly, in the cuprate systems, the extended Drude mode is relatively well isolated in a low-energy region because all of the Lorentz modes of the system are located in the high-energy region above $\sim 2 \mathrm{eV}^{42}$. By contrast, in Fe-pnictide systems, the extended Drude mode significantly overlaps with Lorentz modes located in the low-energy region ${ }^{50}$ (also see Fig. 2(b) and Fig. 3). Therefore, the optical self-energy is relatively better defined in cuprate systems than in Fe-pnictides. We note that both systems can be reasonably well analyzed to reveal the correlation effects by using the extended Drude-Lorentz model. It is worthwhile to note that, in our analysis, we assumed that no interband transitions exist below 200 meV other than tails of the Lorentz modes located at the higher energy. If there is a sharp and strong Lorentz mode below $200 \mathrm{meV}$ we expect to be able to observe it because its line shape is different from that of the extended Drude mode ${ }^{50,51}$.

\section{Conclusions}

In conclusion, we developed a new approach for analyzing the optical spectra of correlated electron systems. The new approach was named as the extended Drude-Lorentz model. The extended Drude mode can be obtained from the electron-boson spectral density function using a reverse process ${ }^{26}$. We compared the extended Drude-Lorentz model with the two Drude-Lorentz model. The extended Drude-Lorentz model explicitly provides information on the correlations between charge carriers. We applied this newly developed approach to measured optical conductivity spectra of K-doped Ba-122 single crystal samples at various doping levels in a wide (from underdoped to overdoped) doping region. We obtained the electron-boson spectral density functions $\left(I^{2} B(\omega)\right)$ at the various doping levels. We also obtained doping-dependent mass renormalization factor $(\lambda)$, which exhibits a dome shape. This factor is maximized near optimally doping level. The different doping-dependent behaviors of $I^{2} B(\omega)$ of the two high-temperature superconducting systems (cuprates and Fe-pnictides) might be associated with pseudogaps; we demonstrated that if we include the pseudogap in our model the doping-dependent mass renormalization factors of the two material systems became similar to each other. This newly developed method will be helpful for conceptually understanding of measured optical spectra in the extended Drude-Lorentz model and also useful for analyzing measured optical spectra of strongly correlated electron systems, 
including high-temperature superconductors and heavy fermion systems.

* Electronic address: jungseek@skku.edu

1 Kamihara, Y. et al. Iron-based layered superconductor: LaOFeP. J. Am. Chem. Soc. 128, $10012(2006)$.

2 Lebegue, S. Electronic structure and properties of the fermi surface of the superconductor laofep. Phys. Rev. B 75, 035110 (2007).

3 Ding, H. et al. Observation of fermi-surface-dependent nodeless superconducting gaps in $\mathrm{Ba}_{0.6} \mathrm{~K}_{0} .4 \mathrm{Fe}_{2} \mathrm{As}_{2}$. Europhys. Lett. 83, 47001 (2008).

4 Kondo, T. et al. Momentum dependence of the superconducting gap in $\mathrm{NdFeAsO}_{0.9} \mathrm{~F}_{0.1}$ single crystals measured by angle resolved photoemission spectroscopy. Phys. Rev. Lett. 101, 147003 (2008).

5 Mazin, I. I., Singh, D. J., Johannes, M. D. \& Du, M. H. Unconventional superconductivity with a sign reversal in the order parameter of $\mathrm{LaFeAsO}_{1-x} \mathrm{~F}_{x}$. Phys. Rev. Lett. 101, 057003 (2008).

6 Subedi, A., Zhang, L., Singh, D. J. \& Du, M. H. Density functional study of FeS, FeSe, and FeTe: Electronic structure, magnetism, phonons, and superconductivity. Phys. Rev. B 78, $134514(2008)$.

$7 \mathrm{Wu}$, D. et al. Nodes in the order parameter of superconducting iron pnictides investigated by infrared spectroscopy. Phys. Rev. B 82, 184527 (2010).

8 van Heumen, E. et al. Optical properties of $\mathrm{BaFe}_{2-x} \mathrm{Co}_{x} \mathrm{As}_{2}$. Europhys. Lett. 90, 37005 (2010).

9 Dressel, M., Wu, D., Barisic, N. \& Gorshunov, B. Looking at the superconducting gap of iron pnictides. J. Phys. Chem. Solids 72, 514 (2011).

10 Dai, Y. M. et al. Hidden T-linear scattering rate in $\mathrm{Ba}_{0.6} \mathrm{~K}_{0.4} \mathrm{Fe}_{2} \mathrm{As}_{2}$ revealed by optical spectroscopy. Phys. Rev. Lett. 111, 117001 (2013).

11 Bang, Y. \& Stewart, G. R. Superconducting properties of the $\mathrm{s}^{ \pm}$-wave state: Fe-based superconductors. J. Phys.: Conden. Matter 29, 123003 (2017).

12 Qazilbash, M. M. et al. Electronic correlations in the iron pnictides. Nat. Phys. 5, 647 (2009).

13 Dai, Y. M. et al. Optical conductivity of $\mathrm{Ba}_{0.6} \mathrm{~K}_{0.4} \mathrm{Fe}_{2} \mathrm{As}_{2}$ : The effect of in-plane and out-ofplane doping in the superconducting gap. Europhys. Lett. 104, 47006 (2013).

14 Yang, J. et al. Optical spectroscopy of superconducting $\mathrm{Ba}_{0.55} \mathrm{~K}_{0.45} \mathrm{Fe}_{2} \mathrm{~A}_{2}$ : Evidence for strong 
coupling to low-energy bosons. Phys. Rev. Lett. 102, 187003 (2009).

$15 \mathrm{Wu}, \mathrm{D}$. et al. Eliashberg analysis of optical spectra reveals a strong coupling of charge carriers to spin fluctuations in doped iron-pnictide $\mathrm{BaFe}_{2} \mathrm{As}_{2}$ superconductors. Phys. Rev. B 82, 144519 (2010).

16 Hwang, J., Carbotte, J. P., Min, B. H., Kwon, Y. S. \& Timusk, T. Electron-boson spectral density of lifeas obtained from optical data. J. Phys.: Condens. Matter 27, 055701 (2015).

17 Hwang, J., Timusk, T., Schachinger, E. \& Carbotte, J. P. Evolution of the bosonic spectral density of the high-temperature superconductor $\mathrm{Bi}_{2} \mathrm{Sr}_{2} \mathrm{CaCu}_{2} \mathrm{O}_{8+\delta}$. Phys. Rev. B 75, 144508 (2007).

$18 \mathrm{Tu}$, J. J. et al. Optical properties of the iron arsenic superconductor $\mathrm{BaFe}_{1.85} \mathrm{Co}_{0.15} \mathrm{As}_{2}$. Phys. Rev. B 82, 174509 (2010).

19 D.Wu et al. Optical investigations of the normal and superconducting states reveal two electronic subsystems in iron pnictides. Phys. Rev. B 81, 100512(R) (2010).

20 Min, B. H. et al. Optical properties of the iron-based superconductor lifeas single crystal. New J. of Phys. 15, 073029 (2013).

21 Lee, S. et al. Hidden non-fermi liquid behavior caused by magnetic phase transition in ni-doped ba-122 pnictides. Scientific Reports 5, 12156 (2015).

22 Hwang, J. Electron-boson spectral density function of correlated multiband systems obtained from optical data: $\mathrm{Ba}_{0} \cdot 6 \mathrm{~K}_{0} \cdot 4 \mathrm{Fe}_{2} \mathrm{As}_{2}$ and lifeas. J. Phys.: Condens. Matter 28, 125702 (2016).

23 Hwang, J., Yang, J., Carbotte, J. P. \& Timusk, T. Manifestation of the pseudogap in ab-plane optical characteristics. J. Phys. Condens. Matter 20, 295215 (2008).

24 Puchkov, A. V., Basov, D. N. \& Timusk, T. The pseudogap state in high-T ${ }_{c}$ superconductors: an infrared study. J. Phys.: Cond. Matter 8, 10049 (1996).

25 Hwang, J., Timusk, T. \& Gu, G. D. High-transition-temperature superconductivity in the absence of the magnetic-resonance mode. Nature (London) 427, 714 (2004).

26 Hwang, J. Reverse process of usual optical analysis of boson-exchange superconductors: impurity effects on $s$ - and $d$-wave superconductors. J. Phys.: Condens. Matter 27, 085701 (2015).

$27 \mathrm{Xu}$, Y.-M. et al. Fermi surface dichotomy of the superconducting gap and pseudogap in underdoped pnictides. Nat. Comm. 2, 392 (2011).

28 Moon, S. J. et al. Infrared measurement of the pseudogap of P-doped and Co-doped hightemperature $\mathrm{BaFe}_{2} \mathrm{As}_{2}$ superconductors. Phys. Rev. Lett. 109, 027006 (2012). 
Homes, C. C., Ali, M. N. \& Cava, R. J. Optical properties of the perfectly compensated semimetal $\mathrm{WTe}_{2}$. Phys. Rev. B 92, 161109(R) (2015).

30 Allen, P. B. Electron-phonon effects in the infrared properties of metals. Phys. Rev. B 3, 305 (1971).

31 Shulga, S. V., Dolgov, O. V. \& Maksimov, E. G. Electronic states and optical spectra of HTSC with electron-phonon coupling. Physica C 178, 266 (1991).

32 Hwang, J. Extended drude model analysis of the optical spectra of correlated electron systems in a d-wave superconducting state. Journal of Korean Physical Society 76, 736 (2020).

33 Nakajima, M. et al. Normal-state charge dynamics in doped $\mathrm{BaFe}_{2} \mathrm{As}_{2}$ : Roles of doping and necessary ingredients for superconductivity. Scientific Reports 4, 5873 (2014).

34 Homes, C. C., Reedyk, M. A., Crandles, D. A. \& Timusk, T. Technique for measuring the reflectance of irregular, submillimeter-sized samples. Appl. Opt. 32, 2976 (1993).

35 Wooten, F. Optical Properties of Solids (Academic, New York, 1972). (Note: Key material on page 176).

36 Tanner, D. B. Optical effects in solids (Cambridge Univ. Press, 2019).

37 Wang, N. L. et al. High energy pseudogap and its evolution with doping in fe-based superconductors as revealed by optical spectroscopy. J. Phys.: Conden. Matter 24, 294202 (2012).

38 Marsik, P. et al. Low-energy interband transitions in the infrared response of $\mathrm{Ba}\left(\mathrm{Fe}_{1-x} \mathrm{Co}_{x}\right)_{2} \mathrm{As}_{2}$. Phys. Rev. B 88, 180508 (2013).

$39 \mathrm{Hu}, \mathrm{W}$. , Zhang, Q. \& Wang, N. Optical and raman spectroscopy studies on fe-based superconductors. Physica C 469, 545 (2009).

40 Kim, K. W. et al. Evidence for multiple superconducting gaps in optimally doped $\mathrm{BaFe}_{1.87} \mathrm{Co}_{0.13} \mathrm{As}_{2}$ from infrared spectroscopy. Phys. Rev. B 81, 214508 (2010).

41 Yang, J. et al. Exchange boson dynamics in cuprates: Optical conductivity of $\mathrm{HgBa}_{2} \mathrm{CuO}_{4+\delta}$. Phys. Rev. Lett. 102, 027003 (2009).

42 Hwang, J., Timusk, T. \& Gu, G. D. Doping dependent optical properties of $\mathrm{Bi}_{2} \mathrm{Sr}_{2} \mathrm{CaCu}_{2} \mathrm{O}_{8+\delta}$. J. Phys.: Condens. Matter 19, 125208 (2007).

43 Hashimoto, K. et al. A sharp peak of the zero-temperature penetration depth at optimal composition in $\mathrm{BaFe}_{2}\left(\mathrm{As}_{1-x} \mathrm{P}_{x}\right)_{2}$. Science 336, 1554 (2012).

44 Shimojima, T. et al. Pseudogap formation above the superconducting dome in iron pnictides. Phys. Rev. B 89, 045101 (2014). 
45 Moon, S. J. et al. Infrared pseudogap in cuprate and pnictide high-temperature superconductors. Phys. Rev. B 90, 014503 (2014).

46 Hwang, J. \& Carbotte, J. P. Determination of boson spectrum from optical data in pseudogap phase of underdoped cuprates. Phys. Rev. B 86, 094502 (2012).

47 Sharapov, S. G. \& Carbotte, J. P. Effects of energy dependence in the quasiparticle density of states on far-infrared absorption in the pseudogap state. Phys. Rev. B 72, 134506 (2005).

48 Hwang, J., Carbotte, J. P. \& Timusk, T. Fermi surface arcs and the infrared conductivity of underdoped $\mathrm{YBa}_{2} \mathrm{Cu}_{3} \mathrm{O}_{6.5}$. Euro. Phys. Lett. 82, 27002 (2008).

49 Hwang, J., Carbotte, J. P. \& Timusk, T. Evidence for a pseudogap in underdoped $\mathrm{Bi}_{2} \mathrm{Sr}_{2} \mathrm{CaCu}_{2} \mathrm{O}_{8+\delta}$ and $\mathrm{YBa}_{2} \mathrm{Cu}_{3} \mathrm{O}_{6} .50$ from in-plane optical conductivity measurements. Phys. Rev. Lett. 100, 177005 (2008).

50 Benfatto, L., Cappelluti, E., Ortenzi, L. \& Boeri, L. Extended drude model and role of interband transitions in the midinfrared spectra of pnictides. Phys. Rev. B 83, 224514 (2011).

51 Hwang, J. Low-energy interband transition effects on extended drude model analysis of optical data of correlated electron system. Prog. in Supercon. Cryogen. 21, 6 (2019). 


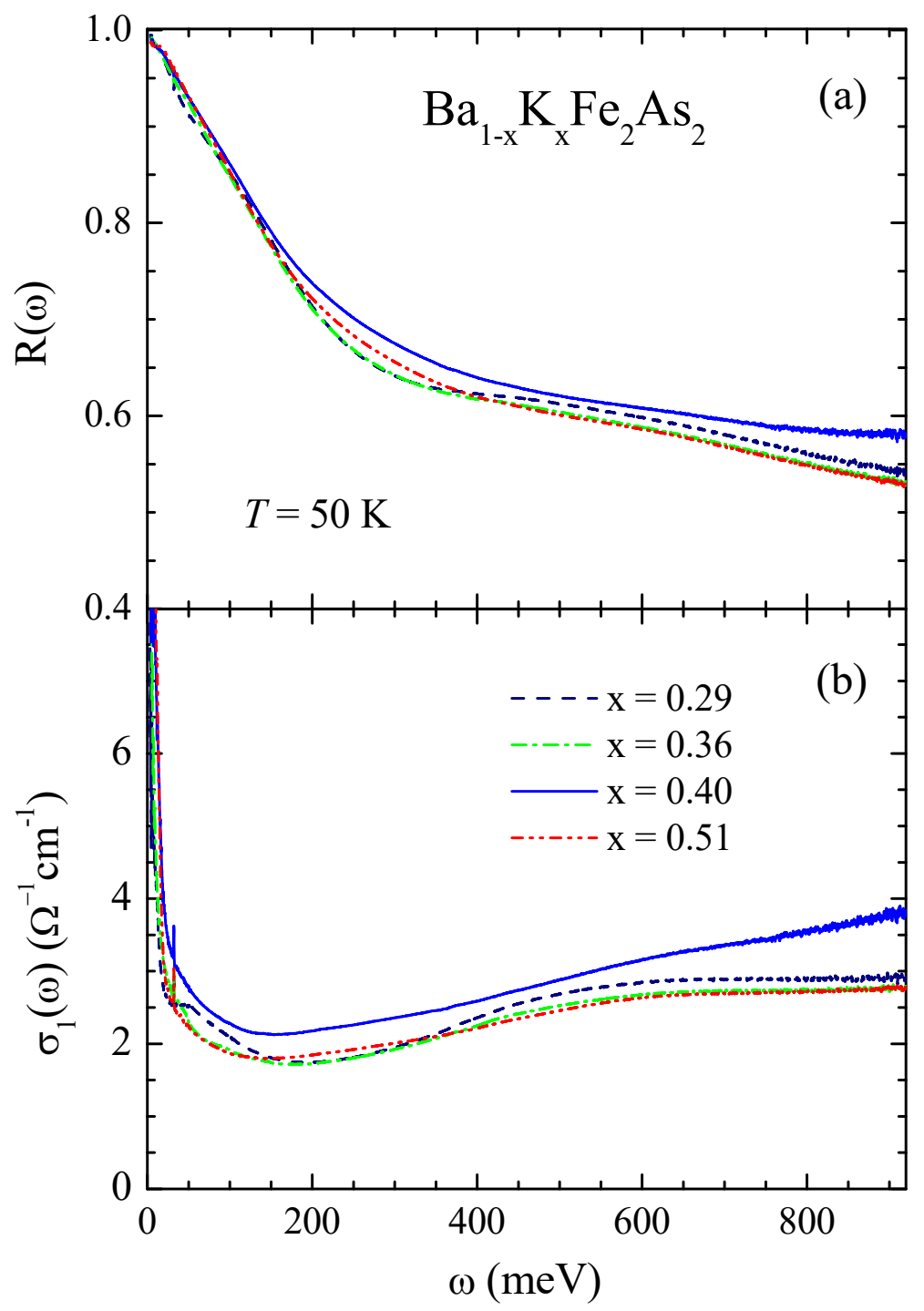

FIG. 1: (Color online) Measured reflectance and optical conductivity spectra at $T=50 \mathrm{~K}$. (a) Measured reflectance spectra of four K-doped Ba-122 single crystal samples. (b) The corresponding optical conductivity spectra obtained using the Kramers-Kronig analysis. 

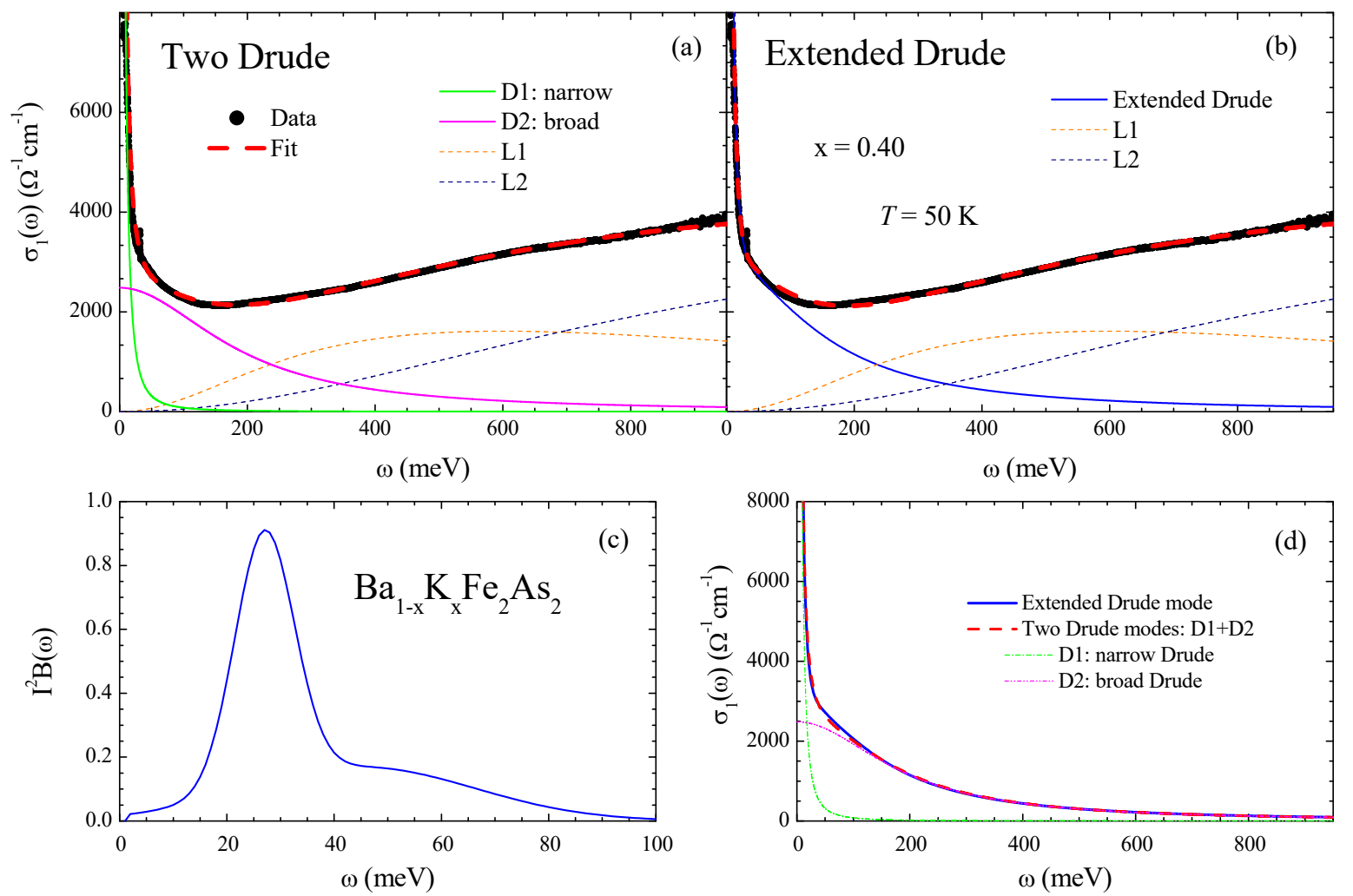

FIG. 2: (Color online) Comparison of results obtained via both the TD-Lorentz and the EDLorentz model analyses. (a) Data and fit obtained by using the TD-Lorentz model. (b) Data and fit obtained by using the ED-Lorentz model. (c) The electron-boson spectral density function obtained by the ED-Lorentz model analysis. (d) Comparison of the optical conductivity of the extended Drude mode with the combined optical conductivity of the two Drude modes. 


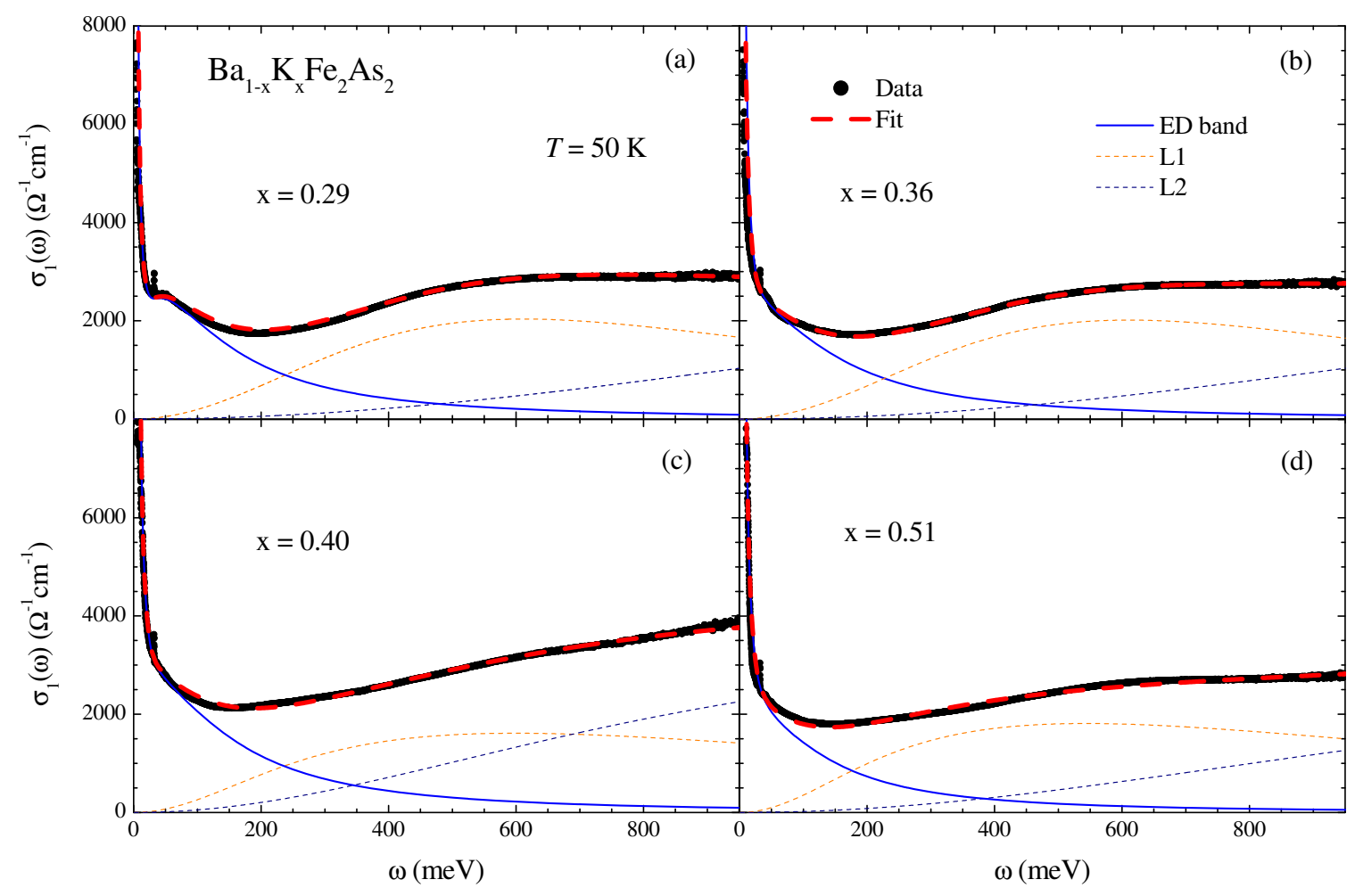

FIG. 3: (Color online) Data and ED-Lorentz model fits of four K-doped Ba-122 $\left(\mathrm{Ba}_{1-x} \mathrm{~K}_{x} \mathrm{Fe}_{2} \mathrm{As}_{2}\right)$ samples at $50 \mathrm{~K}$ : (a) $x=0.29$, (b) $x=0.36$, (c) $x=0.40$, and (d) $x=0.51$. 


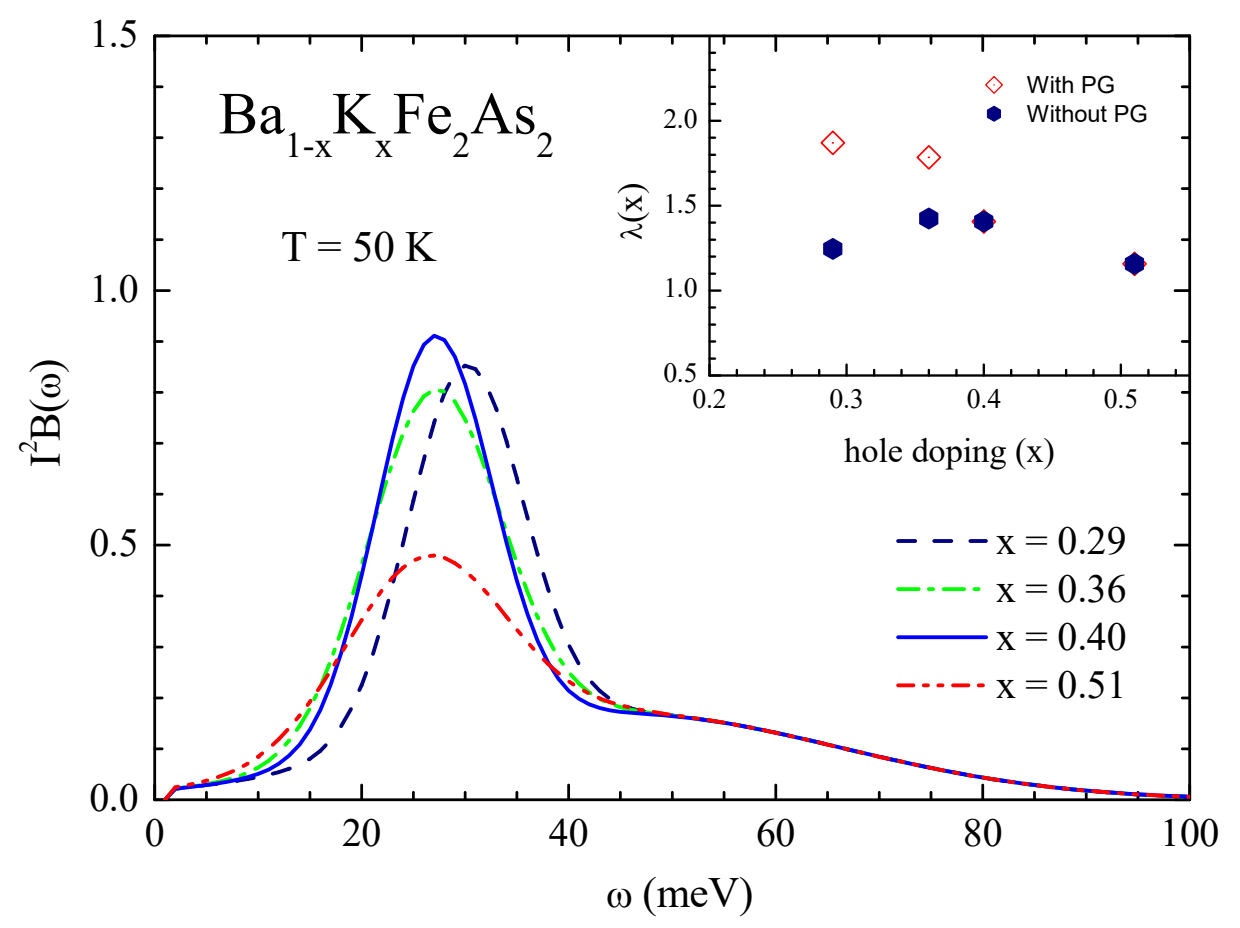

FIG. 4: (Color online) The extracted electron-boson spectral density function, $I^{2} B(\omega)$ of our four samples. In the inset, the mass renormalization factors of the two cases with (open diamond) and without (solid hexagon) including the pseudogaps (PG) are shown. 


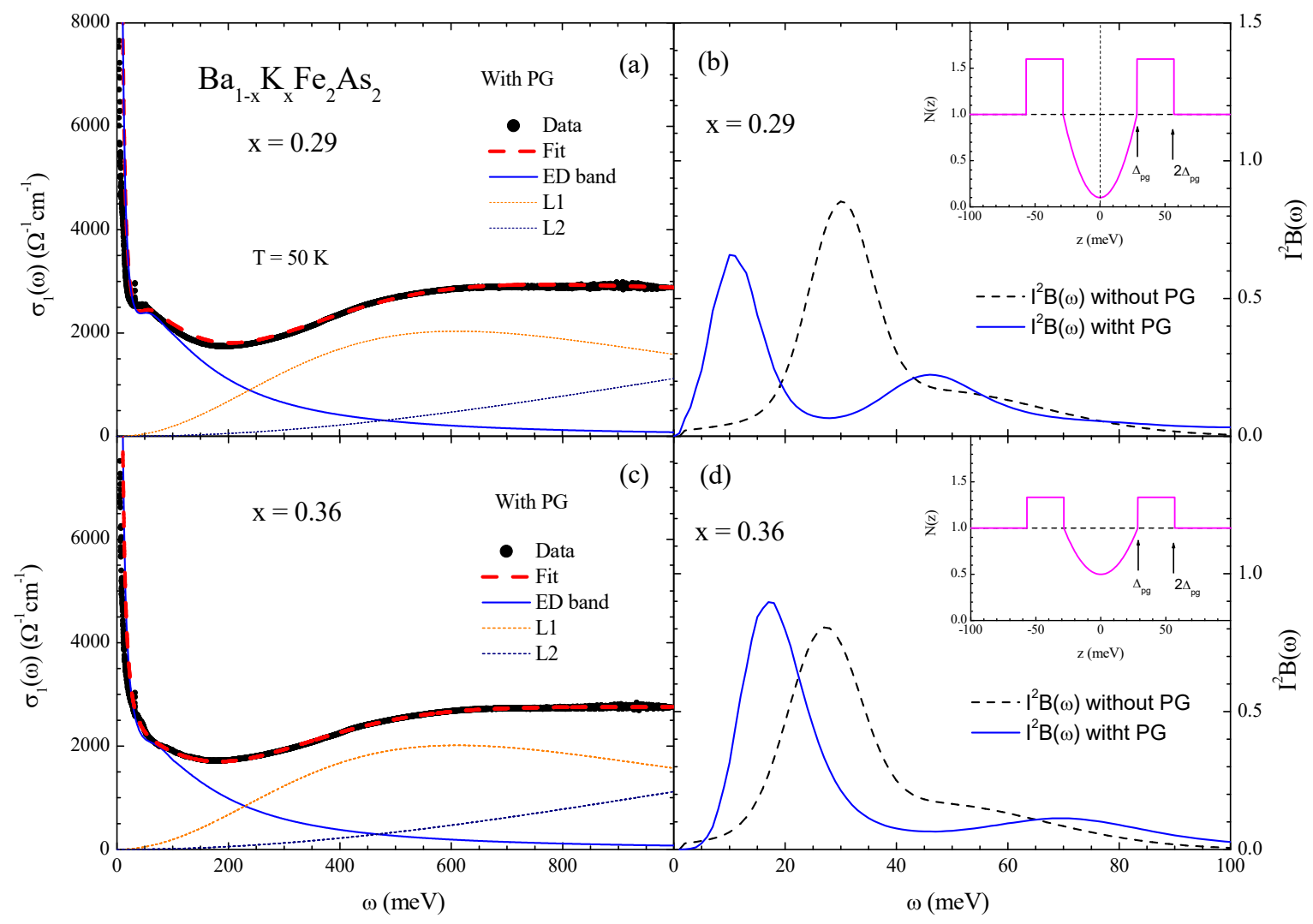

FIG. 5: (Color online) Data and ED-Lorentz model fits with including the pseudogaps. (a) and (c) Data and fits of the two underdoped samples with $x=0.29$ and 0.36 . (b) and (d) The extracted electron-boson spectral density function, $I^{2} B(\omega)$ of the two underdoped samples with the pseudogaps (solid lines) and without pseudogaps (dashed lines). In the insets, the model pseudogaps are displayed. 
Author Contributions J.H. and S.L. wrote the main manuscript, S.L. took the optical data and analyzed them. D.S and H.E. grew and provided the single crystal samples, Y.S. and S.R. contributed to the taking and analyzing of the optical data. All authors reviewed the manuscript.

Acknowledgements JH acknowledges financial support from the National Research Foundation of Korea (NRFK Grant No. 2019R1A6A1007307912, 2020R1A4A4078780, and 2021R1A2C101109811).

Competing Interests The authors declare that they have no competing financial interests.

Correspondence Correspondence and requests for materials should be addressed to Jungseek Hwang (email: jungseek@skku.edu). 